\title{
Detailed Abundances of Planet-Hosting Wide Binaries. II. HD 80606+HD 80607
}

\author{
Claude E. Mack III ${ }^{1}$, Keivan G. Stassun ${ }^{1,2}$, Simon C. Schuler ${ }^{3}$, Leslie Hebb ${ }^{4}$, Joshua A. Pepper ${ }^{5}$
}

\begin{abstract}
We present a detailed chemical abundance analysis of 15 elements in the planet-hosting wide binary system HD 80606 + HD 80607 using Keck/HIRES spectra. As in our previous analysis of the planet-hosting wide binary HD 20782 + HD 20781, we presume that these two G5 dwarf stars formed together and therefore had identical primordial abundances. In this binary, HD 80606 hosts an eccentric $(e \approx 0.93)$ giant planet at $\sim 0.5 \mathrm{AU}$, but HD 80607 has no detected planets. If close-in giant planets on eccentric orbits are efficient at scattering rocky planetary material into their host stars, then HD 80606 should show evidence of having accreted rocky material while HD 80607 should not. Here we show that the trends of abundance versus element condensation temperature for HD 80606 and HD 80607 are statistically indistinguishable, corroborating the recent result of Saffe et al. This could suggest that both stars accreted similar amounts of rocky material; indeed, our model for the chemical signature of rocky planet accretion indicates that HD 80606 could have accreted up to $2.5 M_{\oplus}$ of rocky material - about half that contained in the Solar System and primordial asteroid belt-relative to HD 80607 and still be consistent with the data. Since HD 80607 has no known giant planets that might have pushed rocky planet material via migration onto that star, we consider it more likely that HD 80606/07 experienced essentially no rocky planet accretion. This in turn suggests that the migration history of the HD 80606 giant planet must have been such that it ejected any close-in planetary material that might have otherwise been shepherded onto the star.
\end{abstract}

Subject headings: stars: planetary systems — stars: abundances — stars: binaries: visual stars: individual (HD 80606, HD 80607)

\section{Introduction}

This paper is the second in a series of papers that aims to investigate the relationship between the architectures of planetary systems and the detailed chemical composition of the host star by directly comparing the chemical abundances of each stellar pair in planet-hosting wide binaries (PHWB). In the first paper (Mack et al. 2014), we analyzed the detailed chemical abundances of the PHWB HD $20782+$ HD 20781. For both stars in that system, we found a trend between the elemental abundances $([\mathrm{X} / \mathrm{H}])$ and the elemental

\footnotetext{
${ }^{1}$ Vanderbilt University, Department of Physics \& Astronomy, Nashville, TN 37235 USA

${ }^{2}$ Fisk University, Physics Department, Nashville, TN 37208 USA; keivan.stassun@vanderbilt.edu

${ }^{3}$ University of Tampa, Department of Chemistry, Biochemistry, and Physics, Tampa, FL 33606 USA

${ }^{4}$ Hobart and William Smith Colleges, Department of Physics, Geneva, NY 14456 USA

${ }^{5}$ Lehigh University, Department of Physics, Bethlehem, PA 18015 USA
} 
condensation temperature $\left(T_{\mathrm{C}}\right)$ that, according to a simple model for the accretion of $\mathrm{H}$-depleted rocky planetary material by a solar-type star, was consistent with the ingestion of 10-20 $M_{\oplus}$ by each star.

In this paper, we present the analysis of the detailed abundance trends in the two stars comprising the HD 80606/07 system. HD 80606 and HD 80607 are a common proper motion wide binary with an angular separation of $20.6^{\prime \prime}$ and a projected physical separation of $\sim 1200$ AU (Raghavan et al. 2006). They are both solar-type stars with the same spectral type, G5V, and and apparent V magnitudes of 9.06 and 9.17, respectively (Kharchenko 2001; ESA 1997). HD 80606 hosts a $\sim 4 M_{\text {Jup }}$ on a very eccentric $(e \sim 0.93)$ orbit at $\sim 0.5$ AU (Naef et al. 2001; Pont et al. 2009). On the other hand, there are no detected planetary-mass companions to HD 80607.

If the presence of a close-in eccentric giant planet is an indicator that the host star has likely ingested a significant amount of H-depleted material, as may be the case with HD 20782/81 and a subset of single stars analyzed by Schuler et al. (2011a), then one might expect that the chemical abundances of HD 80606 might show evidence of rocky planetary accretion, while HD 80607 would not. If the chemical abundances of HD 80606/07 are not found to be distinct as was the case in the recent study performed by Saffe et al. (2015), then this suggests that there are other factors besides the present-day architecture of the planetary system that determine the amount of $\mathrm{H}$-depleted rocky material that is ingested by a planet-hosting star. One of these factors might be the specific migration history of the giant planets in the system. As a result, not all host stars with close-in giant planets would necessarily exhibit the abundance trends seen in HD 20782/81. Indeed, the degree of the correlation between planetary-system architecture and the chemical composition of the host star is the main goal of this ongoing series of papers. If a correlation is discovered, then while we cannot expect it to be true for each specific system, it could still serve as tool for more targeted searches for Solar System analogs in general.

In Section 2, we describe our observations and spectral analysis. In Section 3, we present the main results, In Section 4 we discuss the results in the context of a simple model for how the accretion of Earthlike rocky planets would affect refractory elemental abundances as a function of $T_{\mathrm{C}}$, as well as the results of numerical simulations found in the literature, that investigation how giant planet migration affects planetary material interior to the orbit of the giant. Finally, in Section 5 we briefly summarize the main conclusions.

\section{Data and Analysis}

For both HD 80606/07, on UT 2011 Mar 14 we obtained high-resolution, high-signal-to-noise ratio (SNR) spectra with the 10-m Keck I telescope and the HIRES echelle spectrograph (Vogt et al. 1994) in the $R=\lambda / \Delta \lambda=72,000$ mode. We used the kv418 filter with the B2 slit setting $\left(0.574^{\prime \prime} \times 7^{\prime \prime}\right)$ and $2 \times 1$ binning (spatial $\times$ dispersion). The spectra cover a wavelength range from $\sim 3500-9500 \AA$. For each of the stars in the system, one exposure was taken with an integration time of 900s. The SNR in the continuum region near $\lambda 6700$ of $\sim 300$ for HD 80607 and $\sim 330$ for HD 80606. The data were reduced using the MAKEE data reduction routines. A sample spectrum spanning the wavelength region $\lambda 6135-\lambda 6175$ is shown in Figure 1 We note that these spectra were also used by Saffe et al. (2015) for their abundance analysis of these stars. We were unable to obtain a solar spectrum during the same observing run that we observed HD 80606/07. Therefore, for the derivation of the relative chemical abundances, we used a previous Keck/HIRES solar spectrum that we obtained in 2010 June. The solar spectrum has an SNR of $\sim 800$ near $\lambda 6700$.

For both HD 80606/07, chemical abundances relative to solar have been derived from the observed

spectra for 15 elements. We used the 2010 version of the LTE spectral analysis package MOOG (Sneden 
1973) abundances were derived from measurements of the equivalent widths (EWs) of atomic lines using the SPECTRE analysis package (Fitzpatrick \& Sneden 1987). Stellar parameters were obtained by requiring excitation and ionization balance of the Fe I and Fe II lines. The atomic excitation energies $(\chi)$ and transition probabilities $(\log g f)$ were taken from the Vienna Atomic Line Database (VALD; Piskunov et al. 1995; Kupka et al. 1999).

For the odd-Z elements V, Mn, and Co, in order to take into account hyper-fine structure (hfs) effects (Prochaska \& McWilliam 2000), spectral synthesis incorporating hfs components has been used to compare with the EW-based abundances. The hfs components for these elements were obtained from Johnson et al. (2006), and the line lists for wavelength regions encompassing each feature were taken from VALD. The adopted V, Mn, and Co abundances are derived from the hfs analysis and those lines with EWs that are less affected by hfs.

The abundance and error analyses for all elements are described in greater detail in Schuler et al. (2011a). The analysis we performed was nearly identical to our analysis of the PHWB HD 20782/81 (Mack et al. 2014). The stellar parameters and relative abundances of HD 80606/07 are summarized in Table 1. The adopted line list, EWs, and the $\log (N)$ line-by-line abundances of each element for HD 80606/07 and the Sun are given in Table 3 .

\section{Results}

The stellar parameters (Table 1) determined for HD 80606/07 are consistent with both stars having a $\sim$ G5V spectral type. The two stars are essentially stellar twins, and the differences in the stellar parameters are on the order of the $1-\sigma$ uncertainties: $\Delta T_{\text {eff }}=52 \pm 62 \mathrm{~K}, \Delta \log g=0.04 \pm 0.10 \mathrm{dex}$, and $\Delta \xi=0.10 \pm 0.10$ $\mathrm{km} \mathrm{s}^{-1}$. The measured abundances relative to solar of the 15 individual elements that were analyzed are summarized in Table 1

In addition, the stellar parameters of HD 80606/07 are similar enough that we can perform a direct line-by-line differential abundance analysis without using the Sun as a reference. Here, we may compare the stars' abundances differences relative to the statistical errors alone, since the systematic uncertainties in the absolute abundance scale arising from the stellar parameters are eliminated by virtue of the stellar parameters being nearly identical. In Figure 2, for each element, the difference in the means of the line-byline abundances relative to solar are plotted. Averaging over all the elements measured, the mean abundance difference is $-0.018 \pm 0.004$ dex (HD 80606 - HD 80607), where the error is the uncertainty of the mean. In other words, the giant-planet hosting star (HD 80606) is slightly but significantly less enhanced in metals than the non-planet hosting star (HD 80607).

As discussed in detail in Section 4.1, when we compare this difference in the abundances of HD 80606HD 80607 to the differential abundances predicted by our simple accretion model (Mack et al. 2014), we find that we can readily exclude a scenario in which HD 80606 could have accreted $10 M_{\oplus}$ of Earth-like material with respect to HD 80607. A scenario in which HD 80606 accreted $5 M_{\oplus}$ relative to HD 80607 is also strongly statistically ruled out, though we note that two of the elements ( $\mathrm{Al}$ and $\mathrm{Si}$ ) are not excluded by the model. However, these two elements notwithstanding, it appears that we may exclude at the $\sim 5 \sigma$ level a scenario in which as little as $\sim 2.5 M_{\oplus}$ of Earth-like material was accreted by the planet-hosting HD 80606 relative to the non-planet hosting HD 80607. We discuss this result further in Section 4.1 .

For comparison to previous studies of planet-hosting wide binaries, we also determine how the HD 80606/07 
abundances relative to solar $([\mathrm{X} / \mathrm{H}])$ vary as a function of elemental condensation temperature $\left(T_{\mathrm{C}}\right)$. We performed both unweighted and weighted linear fits (since both types of fits have appeared in the literature) to the $[\mathrm{X} / \mathrm{H}]$ versus $T_{\mathrm{C}}$ abundance relations to investigate possible correlations. The numerical values of the slopes derived from these fits are summarized in Table 2. Figure 4 shows the result of the weighted linear fit to the elemental abundances of HD 80606/07 versus $T_{\mathrm{C}}$. The condensation temperatures were taken from the $50 \% T_{\mathrm{C}}$ values listed in Lodders (2003). Note that the $T_{\mathrm{C}}$ values from Lodders (2003) are strictly speaking for solar system composition, whereas HD 80606/07 is metal rich with respect to solar; it is not known whether this introduces any significant additional uncertainty to the $T_{\mathrm{C}}$ values. For both the unweighted and weighted linear fits, the slopes of HD 80606/07 agree within 1- $\sigma$, as they should given that we find the stars to be chemically identical within our uncertainties. Moreover, within 2- $\sigma$, both the unweighted and weighted slopes for both stars are consistent with zero, except for the weighted slope of HD 80606, which differs from zero by more than $4-\sigma\left(\right.$ slope $=(-0.92 \pm 0.21) \times 10^{-5}$ dex $\left.\mathrm{K}^{-1}\right)$. The slightly negative slope of HD 80606 indicates that it may be slightly depleted in refractory elements relative to solar.

In summary, given that the stellar parameters for HD 80606/07 agree within $\sim 1 \sigma$ (including both being overall very metal rich), HD 80606/07 are physically similar enough that they may be considered stellar twins. The most important distinction between these twins is that HD 80606 is known to host an eccentric giant planet whereas HD 80607 is not known to host planets. The $[\mathrm{X} / \mathrm{H}]-T_{\mathrm{C}}$ slopes for HD 80606/07 are identical at the $1 \sigma$ level. However, the line-by-line differential abundances for each element reveal that the planet-hosting HD 80606 is slightly but significantly less enhanced in metals (Table 5), and that a scenario in which HD 80606 has accreted more than $\sim 2.5 M_{\oplus}$ of Earth-like material relative to HD 80607 can be excluded to high statistical significance.

\section{Discussion}

Building on our previous work (Mack et al. 2014), we again interpret our results in terms of a simple model for the accretion of Earth-like material by a Sun-like star. As has been suggested in both Mack et al. (2014) and Schuler et al. (2011a), stars with close-in giant planets may be more likely to accrete significant amounts of rocky planetary material. This accretion, if it occurs, may be the result of the giant planet scattering rocky planets or planetesimals into the star as it migrates inward. Given that HD 80606 hosts a close-in giant planet, while HD 80607 does not, and that HD 80606/07 can be considered stellar twins, then the HD 80606/07 system is an ideal laboratory for investigating if rocky-planet accretion triggered by giant-planet migration occurred in this particular system. Furthermore, systems like HD 80606/07 will serve as benchmark members of the growing catalog of planet-hosting wide binaries that can be used to determine the occurrence rate of rocky-planet accretion.

\subsection{Upper limits on the amount of rocky planetary material scattered into HD 80606/07}

In our analysis of HD 20782/81 (Mack et al. 2014), we created a simple model for the impact that the accretion of a rocky planet would have on the atmospheric composition of a solar-type star. Our discussion in this section refers heavily to that model; we direct the reader to Mack et al. (2014) for details and here describe the modifications relevant to the current analysis. In particular, because the bulk compositions of HD 20782/81 $\left([\mathrm{Fe} / \mathrm{H}]_{82}=-0.02\right.$ and $\left.[\mathrm{Fe} / \mathrm{H}]_{81}=+0.04\right)$ are consistent with solar, we were able to assume in Mack et al. (2014) that their primordial compositions were approximately equal to the present-day solar 
composition. As a result, we were able to use literature values for the composition of the Earth (McDonough 2001) and the composition of the Sun (Asplund et al. 2009) to estimate how the accretion of Earth-like rocky material would alter the photospheric abundances of a Sun-like star. Under the assumptions of our model, the $[\mathrm{X} / \mathrm{H}]-T_{\mathrm{C}}$ slope for HD 20782 was consistent with the ingestion of $\sim 10 M_{\oplus}$, while the slope for HD 20781 was consistent with the ingestion of $\sim 20 M_{\oplus}$. In both cases, this is the amount of material that HD 20782 or HD 20781 may have accreted with respect to the Sun. With respect to each other, their $[\mathrm{X} / \mathrm{H}]-T_{\mathrm{C}}$ slopes are consistent within 1- $\sigma$.

In the case of the present analysis, since the bulk compositions of HD 80606/07 $\left([\mathrm{Fe} / \mathrm{H}]_{06}=+0.35\right.$ and $\left.[\mathrm{Fe} / \mathrm{H}]_{07}=+0.35\right)$ are not consistent with solar, and are in fact quite metal-rich relative to the Sun, we need to modify our model accordingly. Instead of assuming the primordial composition of HD 80606/07 was consistent with solar, we assume that the primordial composition of HD 80606/07 was consistent with the present-day composition of HD 80607. This assumption is motivated by our previous work (Mack et al. 2014) and (Schuler et al. 2011a) that indicated that host stars with close-in ( $\lesssim 1$ AU) giant planets may exhibit $[\mathrm{X} / \mathrm{H}]-T_{\mathrm{C}}$ slopes that are consistent with the accretion of rocky planetary material. Therefore, since HD 80606 hosts the close-in giant planet, we presume that the present-day abundances of HD 80607 are more likely to reflect the primordial compositions of both stars.

In modifying our model so that the primordial composition is consistent with HD 80607, we begin with the same approach described in Mack et al. (2014). Namely, we determine the abundance of each element in a given amount of rocky material (in $M_{\oplus}$ ) and in the convection zone of a star with a given mass (in $\left.M_{\odot}\right)$. The rocky material abundance is then added to the convection zone abundance to give a measure of how much the photospheric abundance of a given element would increase as a result of accreting a rocky planet. By varying the amount of rocky material in the model, we can simulate the accretion of rocky planets of varying masses. Even though HD 80606/07 are quite metal-rich, given their stellar parameters we can use the relations described in Torres et al. (2010) to estimate their masses, which are consistent with solar. Pinsonneault et al. (2001) showed that for stars with $T_{\text {eff }}=5500-5600 \mathrm{~K}$, the mass in the convection zone ranges from $\sim 0.03-0.04 M_{\odot}$ (here we use the most metal-rich models considered by those authors, $[\mathrm{Fe} / \mathrm{H}]=+0.2$; however, as those authors demonstrate, the effect on the convection zone depth of changing the metallicity by $\sim 0.1$ dex is negligible). Therefore, in our model, it is a reasonable approximation to set the mass of HD 80607 to the mass of the Sun, and the mass of the convection zone in HD 80607 to the mass of the solar convection zone.

The results of simulating the accretion of $5 M_{\oplus}$ and $20 M_{\oplus}$ by HD 80607 are shown in Figure 5 Like the planetary accretion model for solar-type stars in Mack et al. (2014), the accretion model for metal-rich stars like HD 80607 also predicts that the accretion of rocky planetary material with Earth-like composition tends to create a more positive correlation between $[\mathrm{X} / \mathrm{H}]$ and $T_{\mathrm{C}}$. Increasing the amount of accreted material causes the slopes to become more positive (or equivalently, less negative). Furthermore, when comparing the results to the modified model, we can place upper limits on the amount of each element that could have been accreted by HD 80606 with respect to HD 80607 .

Unlike our analysis of HD 20782/81 (Mack et al. 2014), where we used the abundances relative to solar in order to determine how much rocky material the two stars might have accreted relative to the Sun, with HD 80606/07, the two stars have very similar $T_{\text {eff }}$ so we can directly compare their abundances through a differential analysis. This differential analysis effectively eliminates the systematic uncertainties in our abundance measurements. For this part of the analysis, we employ the means of the line-by-line differential abundances shown in Table 4, as opposed to the difference of the means that are given in Table 2. Since it is the means of the line-by-line differential abundances that are important here, then we use the uncertainty 
in the mean to define our precision. For most of the elements, the uncertainty in the mean is on the order of $\sim 0.01 \mathrm{dex}$, and all of them possess a precision $\lesssim 0.04$ dex.

As a result, within the assumptions of our theoretical model, we can place tight constraints on the amount of material that HD 80606/07 could have accreted with respect to each other on an element-byelement basis. As shown in Figure 2 and discussed in Section 3, we can very securely rule out a scenario in which HD 80606 accreted as much as $10 M_{\oplus}$ of Earth-like material relative to HD 80607, and statistically we can place an upper limit of $\sim 2.5 M_{\oplus}$ (formally $10 \sigma$ ).

This upper limit of $2.5 M_{\oplus}$ is roughly half the amount of rocky material contained in the Solar System terrestrial planets and primordial asteroid belt (e.g. Chambers 2010), and in fact the $[\mathrm{X} / \mathrm{H}]$ versus $T_{\mathrm{C}}$ data are consistent with no rocky accretion at all so the actual amount accreted could have been less than even this. This implies that any primordial close-in rocky material must have been prevented from reaching the stars, as we now discuss.

\subsection{A scenario explaining the lack of a planet accretion signature in HD 80606/07}

An important question in the study of exoplanetary systems is the frequency with which planet-hosting stars accrete rocky planetary material during the formation and migration processes of giant planets. Previous studies of planet-hosting wide binaries with FGK components where at least one component has a close-in giant planet (by "close-in" we mean $\lesssim 1 \mathrm{AU}$ ), as well as FGK single stars with close-in giants, have suggested that close-in giant planets may push significant amounts of inner rocky planetary material into their host stars (Schuler et al. 2011a; Schuler et al. 2011b; Mack et al. 2014; Teske et al. 2015; Biazzo et al. 2015). If this rocky-planet accretion process is a common occurrence, and if the rocky material is distributed evenly over the convection zone and remains there for a significant fraction of the star's main-sequence lifetime, then for solar-type planet-hosting wide binaries where one component is known to host a close-in giant planet and the other is not, the star with the close-in giant planet, on average, may be more likely to have refractory element abundances that are enhanced with respect to its companion.

In the case of HD 80606/07, given the fact that HD 80606 has a very eccentric giant planet that approaches within $\lesssim 0.03 \mathrm{AU}$, it may be surprising that its slope for $[\mathrm{X} / \mathrm{H}]$ versus $T_{\mathrm{C}}$ does not suggest the accretion of rocky planetary material relative to its companion or the Sun, unlike the stars in the wide binary HD 20782/81, which both have close-in eccentric giant planets. Indeed, as we have seen, there is an upper limit of $2.5 M_{\oplus}$ for the amount of rocky material that HD 80606 could have accreted relative to HD 80607.

However, it is important to keep in mind that $2.5 M_{\oplus}$ or more of inner rocky planets may be rare or at least uncommon. The recent discovery of Kepler-444 (Campante et al. 2015) provides an example of a planetary system with five inner rocky planets. Had a migrating giant planet scattered them into the host star, the ingested rocky material may well have been below our current detection threshold of $2.5 M_{\oplus}$ for the HD 80606. In the case of HD 80606, if it had ingested four planets identical in mass to the inner rocky planets in the Solar System (and with our model assumption that the planets have Earth-like composition), we would have been unable to detect the increase in the rocky elemental abundances. Therefore, the question of how often a rocky-planet accretion event occurs for solar-type stars can only truly be answered as a function of accreted mass, and our data for the HD 80606/07 system cannot provide a clear picture for ingested masses below $2.5 M_{\oplus}$. 
Finally, a recent study by Mustill et al. (2015) carried out numerical simulations of giant planets on eccentric orbits with pericenters of $\lesssim 3 \mathrm{AU}$. That study found that not only did these giant planets frequently interact strongly with inner rocky planets, but the giant planet also could in some cases accrete the rocky planets onto themselves. This would lead to giant planets that have cores enriched in heavy elements, such as those studied by Guillot et al. (2006). In this case, it would be interesting to investigate if HD 80606b shows evidence of having an enriched core, which could potentially explain why HD 80606 failed to accrete more than $2.5 M_{\oplus}$ of rocky material relative to HD 80607 .

\subsection{Comparison to Prior Work}

Our results are in good agreement with the recent findings of HD 80606/07 by Saffe et al. (2015). They also found that HD 80606/07 were not depleted in refractory elements. As they note, the lack of a trend with $T_{\mathrm{C}}$ does appear to imply that the existence of a close-in giant planet does not always lead to the ingestion of H-depleted, refractory-rich planetary material by the host star (a conclusion that also agrees with the result from Liu et al. 2014).

Our own analysis extends the Saffe et al. (2015) findings by adding the quantitative model of Mack et al. (2014), which allows us to stringently constrain the amount of rocky material that could have been ingested by the planet-hosting HD 80606 relative to the non-planet-hosting HD 80607: $\lesssim 2.5 M_{\oplus}$. Applying our model to the individual abundance measurements reported by Saffe et al. (2015), we find, given the average differential abundance of $+0.010 \pm 0.019$ dex reported by them, a similar upper limit of $\sim 5 M_{\oplus}(3 \sigma$ confidence) although with less precision than the upper limit derived from our work reported here. In other words, based on the level of differential abundances that can be reliably measured by present-day techniques such as used in Saffe et al. (2015) and in the present study, it remains possible that HD 80606 could have ingested 2.5-5 $M_{\oplus}$ with respect to $\mathrm{HD} 80607$.

At the same time, it remains true that chemical abundance studies of PHWBs with close-in giant planets have found varying results. Some appear to see a clear enhancement of at least a few rocky elements when performing a differential abundance analysis between the two stars in a given PHWB, such as the enhancement in refractory elements of X0-2N with respect to X0-2S reported by Teske et al. (2015), Biazzo et al. (2015) and Ramírez et al. (2015), as well as the enrichment of 16 Cyg B with respect to 16 Cyg A reported by Ramírez et al. (2011) and Tucci Maia et al. (2014). On the other hand, others have found essentially no difference between the chemical abundances of the stars in the PHWB: Saffe et al. (2015) and this present work, the study of HAT-P-1 by Liu et al. (2014), and a separate analysis of the 16 Cyg system performed Schuler et al. (2011b). Also, while not a direct measure of the elemental abundances of the stellar photospheres, asteroseismic modeling of $16 \mathrm{Cyg} \mathrm{A}$ and B suggests that the two stars have the same chemical compositions (Metcalfe et al. 2012, 2015).

These mixed results underscore the need for an analysis of a larger sample of PHWBs and for analysis by multiple investigators using different instruments and analysis techniques. Moreover, increasing the sample size of detailed abundances of PHWBs will facilitate the more precise delineation of possible chemical signatures resulting from the accretion of rocky planet material and planet formation itself, and the disentanglement of the effects of the two processes. Whereas the accretion of H-depleted rocky planet material would be expected to enhance the abundances of refractory elements of a star, it has been suggested that planet formation, rocky planet formation in particular, may indeed deplete refractory abundances of a star (e.g. Meléndez et al. 2009; Ramírez et al. 2009), although not all observational results support this sugges- 
tion (e.g. Adibekyan et al. 2014; Schuler et al. 2015). The available evidence across the various PHWBs that have been analyzed strongly indicates real astrophysical variation in the abundance patterns of stars with planets, and the reasons for the variation, whether due to accretion, planet formation, Galactic chemical evolution, or some other unknown effect, remain unclear.

\section{Summary and Conclusion}

We have used Keck/HIRES observations to perform a detailed spectroscopic analysis of the binary HD 80606/07, in which one star (HD 80606) is known to host a very eccentric $(e \approx 0.93)$ giant planet with closest approach of $\sim 0.03 \mathrm{AU}$, while its sibling (HD 80607) is not known to host planets. We find that the two stars are otherwise "twin" G5 dwarfs, with nearly identical physical properties, and presumably with the same age and initial chemical makeup, making this an unusually good astrophysical laboratory for exploring differences in planet hosting characteristics among otherwise similar stars.

Following our methodology from analysis of another PHWB system (HD 20782/81; Mack et al. 2014), we have performed a detailed chemical abundance analysis of the two stars in HD 80606/07. Similar to the recent results of Saffe et al. (2015), we find linear least-squares fits to the refractory elemental abundances $([\mathrm{X} / \mathrm{H}])$ versus elemental condensation temperature $\left(T_{\mathrm{C}}\right)$ yield slopes that are statistically indistinguishable for the two stars. Evidently, the very different planetary system architectures of the two stars did not result in a difference greater than $\sim 0.03-0.04$ dex in the photospheric elemental abundances of the stars. This finding is in stark contrast to the clearly different $[\mathrm{X} / \mathrm{H}]$ vs. $T_{\mathrm{C}}$ patterns that we found for the two stars in HD 20782/81 (Mack et al. 2014). Indeed, in that analysis we developed a simple model for the pollution of the stellar photospheres by ingestion of small rocky planets, likely previously scattered into the stars by the currently observed giant planets, which could successfully explain the observed chemical abundance patterns and differences between the two stars.

However, when we analyze the abundance differences on a relative, element-by-element basis, thus eliminating potential systematics arising from uncertainties in the stellar parameters, the means of the lineby-line differential abundances $\left([\mathrm{X} / \mathrm{H}]_{06}-[\mathrm{X} / \mathrm{H}]_{07}\right)$ for each element show that the heavy element abundances of HD 80606 are $-0.018 \pm 0.004$ dex relative to those of HD 80607. In the present case of HD 80606/07, our rocky planet accretion model suggests an upper limit of $10 M_{\oplus}$ for the differential amount of rocky planetary material that could have scattered onto HD 80606 over HD 80607 . In fact the observations are consistent with zero rocky planetary accretion. Indeed, even $2.5 M_{\oplus}$ would represent the accretion of about half the amount of rocky material sequestered in the terrestrial planet of the Solar System, plus the primordial asteroid belt. Thus we consider it most likely that HD 80606 accreted little if any rocky planetary material relative to HD 80607. This may seem surprising particularly in the case of HD 80606, given the fact that it hosts a very eccentric giant planet that approaches within $\lesssim 0.03 \mathrm{AU}$, and thus might be expected to very easily sweep inner rocky planets into the star.

However, as we have discussed, if the giant planet in HD 80606 previously migrated inward very quickly, it may have been an inefficient shepherd of material interior to its orbit. We suggest that this idea is plausible given that larger orbital eccentricities are often the result of a more violent migration history, such as planetplanet scattering. In this scenario, we expect that any rocky planets that might have been originally interior to the HD 80606 giant planet would have been preferentially ejected from the system, rather than directed into the host star. 
C.E.M. and K.G.S acknowledge support by NSF AAG AST-1009810 and NSF PAARE AST-0849736. S.C.S. acknowledges support provided by grant NNX12AD19G from the National Aeronautical and Space Administration as part of the Kepler Participating Scientist Program. The data presented herein were obtained at the W.M. Keck Observatory, which is operated as a scientific partnership among the California Institute of Technology, the University of California and the National Aeronautics and Space Administration. The Observatory was made possible by the generous financial support of the W.M. Keck Foundation. The authors wish to recognize and acknowledge the very significant cultural role and reverence that the summit of Mauna Kea has always had within the indigenous Hawaiian community. We are most fortunate to have the opportunity to conduct observations from this mountain.

Facilities: Keck I: HIRES

\section{REFERENCES}

Adibekyan, V. Z., González Hernández, J. I., Delgado Mena, E., et al. 2014, A\&A, 564, L15

Asplund, M., Grevesse, N., Sauval, A. J., \& Scott, P. 2009, ARA\&A, 47, 481

Biazzo, K., Gratton, R., Desidera, S., et al. 2015, arXiv:1506.01614

Campante, T. L., Barclay, T., Swift, J. J., et al. 2015, ApJ, 799, 170

Chambers, J. E. 2010, ApJ, 724, 92

Deal, M., Richard, O., \& Vauclair, S. 2015, arXiv:1509.06958

ESA, ed. 1997, ESA Special Publication, Vol. 1200, The HIPPARCOS and TYCHO catalogues. Astrometric and photometric star catalogues derived from the ESA HIPPARCOS Space Astrometry Mission

Fitzpatrick, M. J., \& Sneden, C. 1987, in Bulletin of the American Astronomical Society, Vol. 19, Bulletin of the American Astronomical Society, 1129

Johnson, J. A., Ivans, I. I., \& Stetson, P. B. 2006, ApJ, 640, 801

Kharchenko, N. V. 2001, Kinematika i Fizika Nebesnykh Tel, 17, 409

Kupka, F., Piskunov, N., Ryabchikova, T. A., Stempels, H. C., \& Weiss, W. W. 1999, A\&AS, 138, 119

Liu, F., Asplund, M., Ramírez, I., Yong, D., \& Meléndez, J. 2014, MNRAS, 442, L51

Lodders, K. 2003, ApJ, 591, 1220

Mack, III, C. E., Schuler, S. C., Stassun, K. G., \& Norris, J. 2014, ApJ, 787, 98

Metcalfe, T. S., Chaplin, W. J., Appourchaux, T., et al. 2012, ApJ, 748, L10

Metcalfe, T. S., Creevey, O. L., \& Davies, G. R. 2015, ApJ, 811, L37

Meléndez, J., Asplund, M., Gustafsson, B., \& Yong, D. 2009, ApJ, 704, L66

McDonough, W. 2001, in The Composition of the Earth, in Earthquake Thermodynamics and Phase Transitions in the Earth's Interior (International Geophysics Series, Vol. 76), ed. R. Teisseyre \& E. Majewski (San Diego, CA: Academic Press) 
Naef, D., Latham, D. W., Mayor, M., et al. 2001, A\&A, 375, L27

Pinsonneault, M. H., DePoy, D. L., \& Coffee, M. 2001, ApJ, 556, L59

Piskunov, N. E., Kupka, F., Ryabchikova, T. A., Weiss, W. W., \& Jeffery, C. S. 1995, A\&AS, 112,525

Pont, F., Hébrard, G., Irwin, J. M., et al. 2009, A\&A, 502, 695

Prochaska, J. X., \& McWilliam, A. 2000, ApJ, 537, L57

Ramírez, I., Khanal, S., Aleo, P., et al. 2015, ApJ, 808, 13

Ramírez, I., Meléndez, J., \& Asplund, M. 2009, A\&A, 508, L17

Ramírez, I., Meléndez, J., Cornejo, D., Roederer, I. U., \& Fish, J. R. 2011, ApJ, 740, 76

Saffe, C., Flores, M., \& Buccino, A. 2015, A\&A, 582, A17

Schuler, S. C., Flateau, D., Cunha, K., et al. 2011a, ApJ, 732, 55

Schuler, S. C., Cunha, K., Smith, V. V., et al. 2011b, ApJ, 737, L32

Schuler, S. C., Vaz, Z. A., Katime Santrich, O. J., et al. 2015, ApJ, 815, 5

Sneden, C. A. 1973, PhD thesis, THE UNIVERSITY OF TEXAS AT AUSTIN.

Teske, J. K., Ghezzi, L., Cunha, K., et al. 2015, ApJ, 801, L10

Torres, G., Andersen, J., \& Giménez, A. 2010, A\&A Rev., 18, 67

Tucci Maia, M., Meléndez, J., \& Ramírez, I. 2014, ApJ, 790, L25

Vogt, S. S., Allen, S. L., Bigelow, B. C., et al. 1994, in Society of Photo-Optical Instrumentation Engineers (SPIE) Conference Series, Vol. 2198, Instrumentation in Astronomy VIII, ed. D. L. Crawford \& E. R. Craine, 362 
Table 1. Stellar Parameters \& Abundances ${ }^{\mathrm{a}}$

\begin{tabular}{lcccc}
\hline \hline & HD 80606 & HD 80607 & HD 80606-HD 80607 & $T_{\mathrm{C}}(\mathrm{K})$ \\
\hline$T_{\text {eff }}(\mathrm{K})$ & $5613 \pm 44$ & $5561 \pm 43$ & $52 \pm 62$ & $\ldots$ \\
$\log g(\mathrm{cgs})$ & $4.43 \pm 0.08$ & $4.47 \pm 0.06$ & $0.04 \pm 0.10$ & $\ldots$ \\
$\xi\left(\mathrm{km} \mathrm{s}{ }^{-1}\right)$ & $1.36 \pm 0.07$ & $1.26 \pm 0.07$ & $0.10 \pm 0.10$ & $\ldots$ \\
{$[\mathrm{Na} / \mathrm{H}] \ldots$} & $+0.45 \pm 0.03 \pm 0.05$ & $+0.46 \pm 0.04 \pm 0.05$ & $-0.01 \pm 0.01 \pm 0.02$ & 958 \\
{$[\mathrm{Mg} / \mathrm{H}]^{\mathrm{d}}$.} & $+0.40 \pm 0.00 \pm 0.03$ & $+0.47 \pm 0.00 \pm 0.03$ & $\ldots$ & 1336 \\
{$[\mathrm{Al} / \mathrm{H}] \ldots$} & $+0.39 \pm 0.01 \pm 0.03$ & $+0.37 \pm 0.02 \pm 0.03$ & $+0.02 \pm 0.01 \pm 0.01$ & 1653 \\
{$[\mathrm{Si} / \mathrm{H}] \ldots$} & $+0.34 \pm 0.01 \pm 0.01$ & $+0.34 \pm 0.01 \pm 0.01$ & $+0.01 \pm 0.01 \pm 0.02$ & 1310 \\
{$[\mathrm{Ca} / \mathrm{H}] \ldots$} & $+0.28 \pm 0.01 \pm 0.05$ & $+0.31 \pm 0.01 \pm 0.04$ & $-0.03 \pm 0.00 \pm 0.01$ & 1517 \\
{$[\mathrm{Sc} / \mathrm{H}] \ldots$} & $+0.36 \pm 0.02 \pm 0.04$ & $+0.39 \pm 0.04 \pm 0.05$ & $-0.03 \pm 0.02 \pm 0.03$ & 1659 \\
{$[\mathrm{Ti} / \mathrm{H}] \ldots$} & $+0.36 \pm 0.02 \pm 0.05$ & $+0.42 \pm 0.02 \pm 0.05$ & $-0.05 \pm 0.02 \pm 0.05$ & 1582 \\
{$[\mathrm{~V} / \mathrm{H}] \ldots$} & $+0.41 \pm 0.01 \pm 0.05$ & $+0.45 \pm 0.02 \pm 0.06$ & $-0.03 \pm 0.01 \pm 0.03$ & 1429 \\
{$[\mathrm{Cr} / \mathrm{H}] \ldots$} & $+0.34 \pm 0.04 \pm 0.06$ & $+0.39 \pm 0.04 \pm 0.05$ & $-0.02 \pm 0.02 \pm 0.04$ & 1296 \\
{$[\mathrm{Mn} / \mathrm{H}]^{\mathrm{d}}$.} & $+0.42 \pm 0.00 \pm 0.06$ & $+0.39 \pm 0.00 \pm 0.06$ & & $\ldots$ \\
{$[\mathrm{Fe} / \mathrm{H}] \ldots$} & $+0.35 \pm 0.01 \pm 0.02$ & $+0.35 \pm 0.01 \pm 0.02$ & $+0.00 \pm 0.01 \pm 0.04$ & 11534 \\
{$[\mathrm{Co} / \mathrm{H}] \ldots$} & $+0.44 \pm 0.01 \pm 0.04$ & $+0.46 \pm 0.01 \pm 0.04$ & $-0.03 \pm 0.01 \pm 0.02$ & 1352 \\
{$[\mathrm{Ni} / \mathrm{H}] \ldots$} & $+0.40 \pm 0.01 \pm 0.03$ & $+0.43 \pm 0.01 \pm 0.03$ & $-0.03 \pm 0.01 \pm 0.03$ & 1353 \\
\hline
\end{tabular}

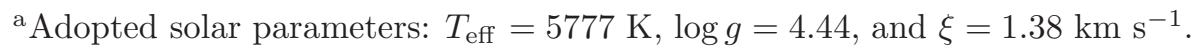

${ }^{\mathrm{b}} \sigma_{\mu}$ - the uncertainty in the mean

${ }^{\mathrm{c}} \sigma_{\text {Total }}-$ quadratic sum of $\sigma_{\mu}$ and uncertainties due to uncertainties in $T_{\text {eff }}, \log g$, and $\xi$.

${ }^{\mathrm{d}}$ In both stars, the abundance measurements for $\mathrm{Mg}$ and $\mathrm{Mn}$ were determined from a single spectral line. That is why the uncertainty in the mean is 0.00 in both stars.

Table 2. Unweighted and Weighted Linear Fits to $[\mathrm{X} / \mathrm{H}]$ vs $T_{\text {eff }}$

\begin{tabular}{|c|c|c|}
\hline & $\begin{array}{l}\text { Unweighted Slope } \\
\quad\left(\operatorname{dex} K^{-1}\right)\end{array}$ & $\begin{array}{l}\text { Weighted Slope } \\
\quad\left(\operatorname{dex} K^{-1}\right)\end{array}$ \\
\hline HD 80606 & $(-11.56 \pm 6.40) \times 10^{-5}$ & $(-0.92 \pm 0.21) \times 10^{-5}$ \\
\hline HD 80607 & $(-8.31 \pm 7.45) \times 10^{-5}$ & $(-0.54 \pm 0.30) \times 10^{-5}$ \\
\hline
\end{tabular}




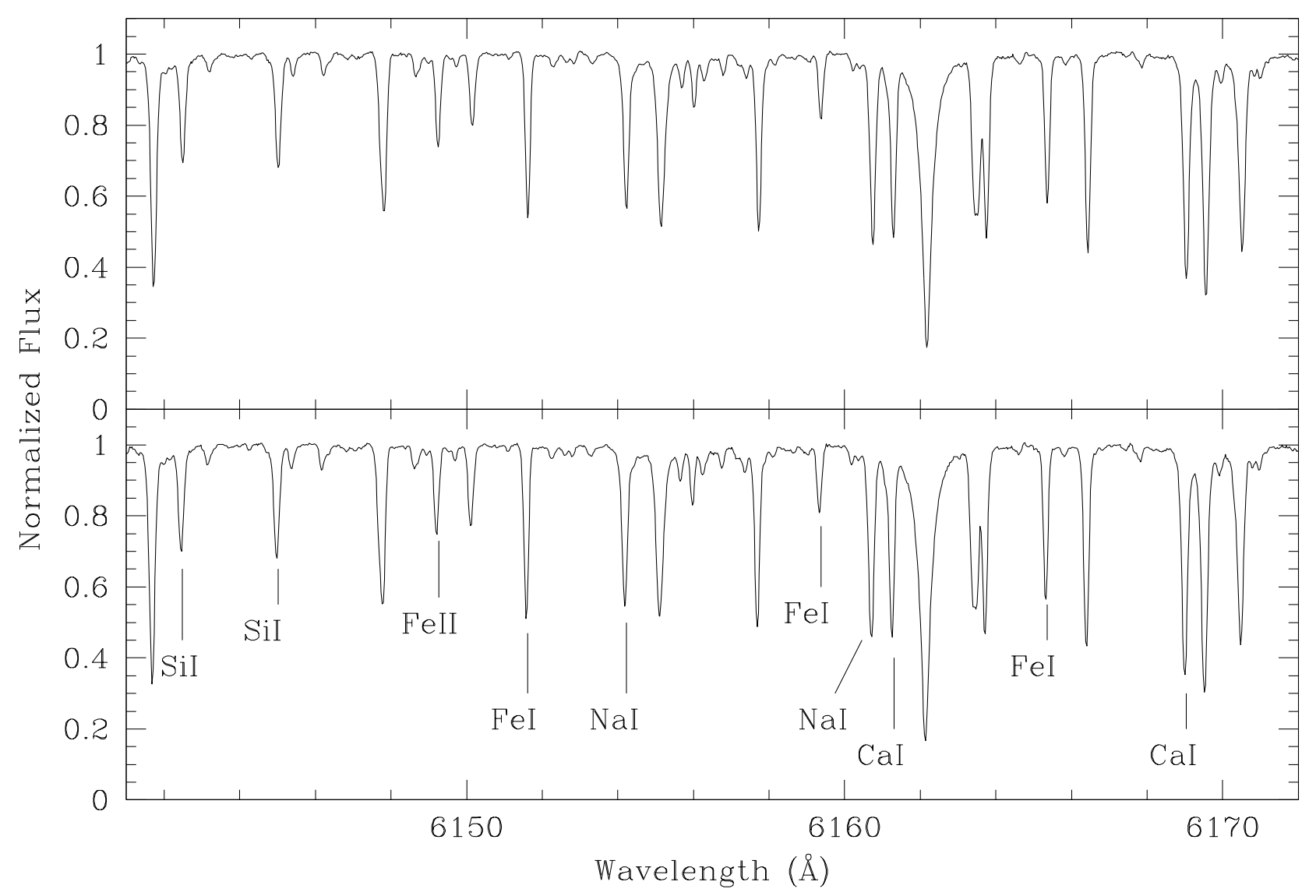

Fig. 1. - Sample Keck/HIRES spectra for HD 80606/07, spanning the wavelength range from $\sim \lambda 6140-$ $\lambda 6170$. The marked lines are a subset of those that were measured. 


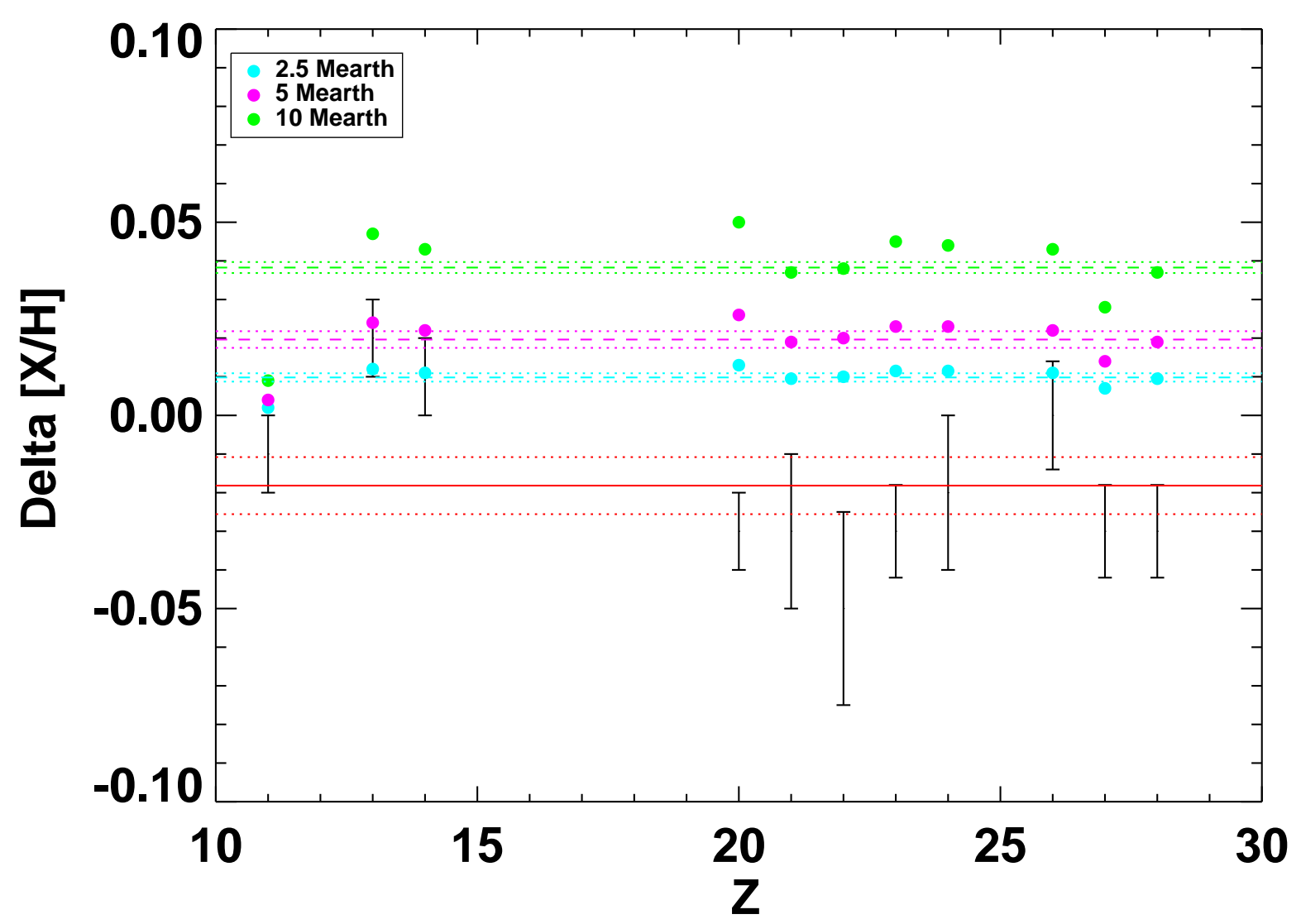

Fig. 2.- Differential abundances for HD $80606-\mathrm{HD} 80607$ as a function of atomic number (Z). The solid red line represents the mean difference of $-0.018 \pm 0.004 \mathrm{dex}$, and the dashed line represent the model predictions of Mack et al. (2014) for different amounts of rocky material ingestion by the planet-hosting HD 80606 relative to non-planet-hosting HD 80607. 


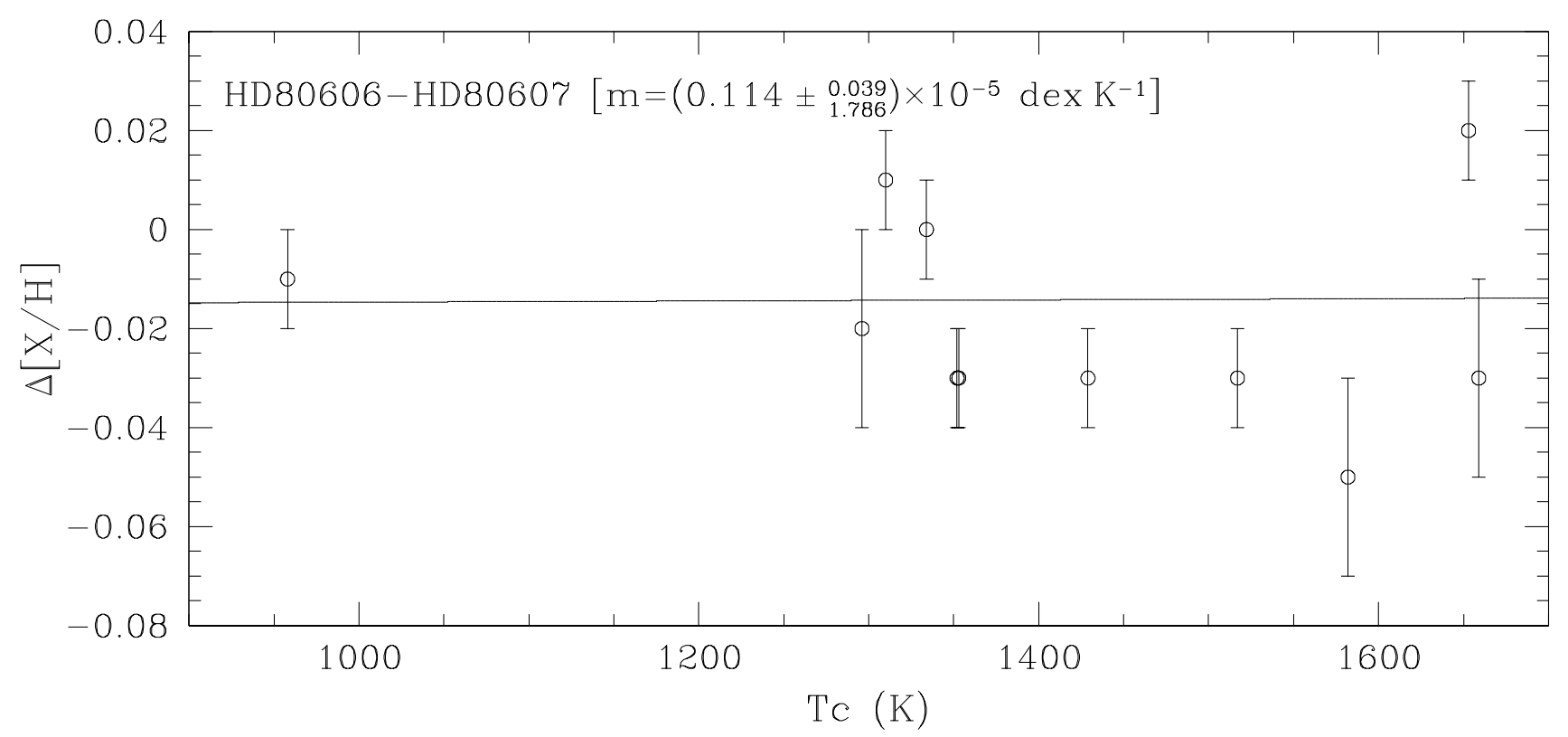

Fig. 3.- Differential abundances for $\mathrm{HD} 80606 / 07$ as a function of condensation temperature $\left(T_{\mathrm{C}}\right)$. 
Table 3. HD 80606/07: Lines Measured, Equivalent Widths, and Abundances

\begin{tabular}{|c|c|c|c|c|c|c|c|c|c|c|c|c|}
\hline \multirow[b]{2}{*}{ Element } & \multirow{2}{*}{$\begin{array}{c}\lambda \\
(\AA)\end{array}$} & \multirow{2}{*}{$\begin{array}{c}\chi \\
(\mathrm{eV})\end{array}$} & \multirow[b]{2}{*}{$\log g f$} & \multirow[b]{2}{*}{$\mathrm{EW} \odot$} & \multirow[b]{2}{*}{$\log N_{\odot}$} & \multirow[b]{2}{*}{$\log N_{\odot, \text { synth }}{ }^{\mathrm{a}}$} & \multicolumn{3}{|c|}{ HD 80606} & \multicolumn{3}{|c|}{ HD 80607} \\
\hline & & & & & & & $\mathrm{EW}$ & $\log N$ & $\log N_{\text {synth }}$ & EW & $\log N$ & $\log N_{\text {synth }}$ \\
\hline $\mathrm{Na} \mathrm{I}$ & 5682.63 & 2.10 & -0.700 & 100.9 & 6.30 & $\ldots$ & 142.9 & 6.70 & $\ldots$ & 146.0 & 6.70 & $\ldots$ \\
\hline $\mathrm{Na} \mathrm{I}$ & 6154.23 & 2.10 & -1.560 & 38.4 & 6.31 & $\ldots$ & 76.5 & 6.77 & $\ldots$ & 80.4 & 6.80 & $\ldots$ \\
\hline $\mathrm{Na} \mathrm{I}$ & 6160.75 & 2.10 & -1.260 & 56.1 & 6.28 & $\cdots$ & 100.6 & 6.78 & $\cdots$ & 102.1 & 6.77 & $\cdots$ \\
\hline $\mathrm{Mg} \mathrm{I}$ & 4730.03 & 4.35 & -2.523 & 67.3 & 7.83 & $\ldots$ & 103.0 & 8.23 & $\ldots$ & 110.2 & 8.30 & $\ldots$ \\
\hline $\mathrm{Al} \mathrm{I}$ & 6696.02 & 3.14 & -1.347 & 38.3 & 6.27 & $\cdots$ & 70.4 & 6.66 & $\ldots$ & 70.8 & 6.65 & $\ldots$ \\
\hline $\mathrm{Al} \mathrm{I}$ & 6698.67 & 3.14 & -1.647 & 22.9 & 6.27 & $\cdots$ & 47.9 & 6.65 & $\cdots$ & 47.9 & 6.62 & $\ldots$ \\
\hline Si I & 5701.10 & 4.93 & -1.581 & 37.2 & 7.08 & $\ldots$ & 59.8 & 7.46 & $\ldots$ & 57.6 & 7.44 & $\ldots$ \\
\hline Si I & 5772.15 & 5.08 & -1.358 & 51.8 & 7.23 & $\ldots$ & 72.6 & 7.56 & $\ldots$ & 71.8 & 7.56 & $\ldots$ \\
\hline Si I & 6125.02 & 5.61 & -1.464 & 30.3 & 7.44 & $\cdots$ & 52.2 & 7.83 & $\cdots$ & 50.5 & 7.81 & $\ldots$ \\
\hline Si I & 6142.48 & 5.62 & -1.295 & 32.4 & 7.31 & $\ldots$ & 52.8 & 7.67 & $\ldots$ & 53.2 & 7.69 & $\ldots$ \\
\hline Si I & 6145.02 & 5.62 & -1.310 & 37.2 & 7.41 & $\cdots$ & 58.4 & 7.76 & $\cdots$ & 56.0 & 7.74 & $\ldots$ \\
\hline Si I & 6243.81 & 5.62 & -1.242 & 47.0 & 7.50 & $\ldots$ & 67.9 & 7.83 & $\ldots$ & 68.2 & 7.84 & $\ldots$ \\
\hline
\end{tabular}

Note. - This table is shown in its entirety on the last three pages of this manuscript (Table 5). A portion is shown here for guidance regarding its form and content.

${ }^{a}$ Indicates the $\log N$ abundance determined from the synthetic fit to a given line. Each synthetic fit was performed with the MOoG synth driver. Synthetic fits were only performed for the subset of V, Mn, and Co lines that were tested for hfs.

${ }^{\mathrm{c}}$ Indicates that the spectral line was tested for hfs. 
Table 4. Comparison of the observed and predicted differential abundances for various amounts of ingested Earth-masses

\begin{tabular}{|c|c|c|c|c|}
\hline \multirow[b]{2}{*}{$\Delta[\mathrm{X} / \mathrm{H}]$} & \multirow{2}{*}{$\begin{array}{c}\text { Observed } \Delta[\mathrm{X} / \mathrm{H}] \\
\text { HD } 80606-\mathrm{HD} 80607\end{array}$} & \multicolumn{3}{|c|}{ (if $\mathrm{HD} 80606 \frac{\text { Predicted } \Delta[\mathrm{X} / \mathrm{H}]}{\text { ingested } x M_{\oplus} \text { w.r.t HD 80607) }}$} \\
\hline & & $2.5 M_{\oplus}$ & $5 M_{\oplus}$ & $10 M_{\oplus}$ \\
\hline$\Delta[\mathrm{Na} / \mathrm{H}]$ & $-0.01 \pm 0.01^{\mathrm{a}}$ & +0.002 & +0.004 & +0.009 \\
\hline$\Delta[\mathrm{Mg} / \mathrm{H}]$ & $\ldots$ & +0.007 & +0.015 & +0.029 \\
\hline$\Delta[\mathrm{Al} / \mathrm{H}]$ & $+0.02 \pm 0.01$ & +0.012 & +0.024 & +0.047 \\
\hline$\Delta[\mathrm{Si} / \mathrm{H}]$ & $+0.01 \pm 0.01$ & +0.011 & +0.022 & +0.043 \\
\hline$\Delta[\mathrm{Ca} / \mathrm{H}]$ & $-0.03 \pm 0.01$ & +0.013 & +0.026 & +0.050 \\
\hline$\Delta[\mathrm{Sc} / \mathrm{H}]$ & $-0.03 \pm 0.02$ & +0.010 & +0.019 & +0.037 \\
\hline$\Delta[\mathrm{Ti} / \mathrm{H}]$ & $-0.05 \pm 0.02$ & +0.010 & +0.020 & +0.038 \\
\hline$\Delta[\mathrm{V} / \mathrm{H}]$ & $-0.03 \pm 0.01$ & +0.011 & +0.023 & +0.045 \\
\hline$\Delta[\mathrm{Cr} / \mathrm{H}]$ & $-0.02 \pm 0.02$ & +0.011 & +0.023 & +0.044 \\
\hline$\Delta[\mathrm{Mn} / \mathrm{H}]$ & $\ldots$ & +0.006 & +0.013 & +0.025 \\
\hline$\Delta[\mathrm{Fe} / \mathrm{H}]$ & $+0.00 \pm 0.01$ & +0.006 & +0.011 & +0.022 \\
\hline$\Delta[\mathrm{Co} / \mathrm{H}]$ & $-0.03 \pm 0.01$ & +0.007 & +0.014 & +0.028 \\
\hline$\Delta[\mathrm{Ni} / \mathrm{H}]$ & $-0.03 \pm 0.01$ & +0.010 & +0.019 & +0.037 \\
\hline
\end{tabular}

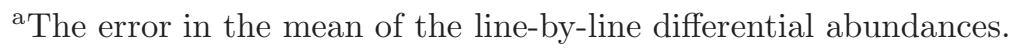

Note. - The predicted $\Delta[\mathrm{X} / \mathrm{H}]$ assumes that the observed chemical composition of HD 80607 is equivalent to the primordial composition of both HD 80606/07. The predicted values show how the abundances of HD 80607 would change after accreting varying amounts $\left(X_{1} M_{\oplus}\right)$ of material with Earth-like composition, i.e., the differential abundance that we would expect if HD 80606 had accreted $X_{1} M_{\oplus}$ while HD 80607 accreted nothing. 


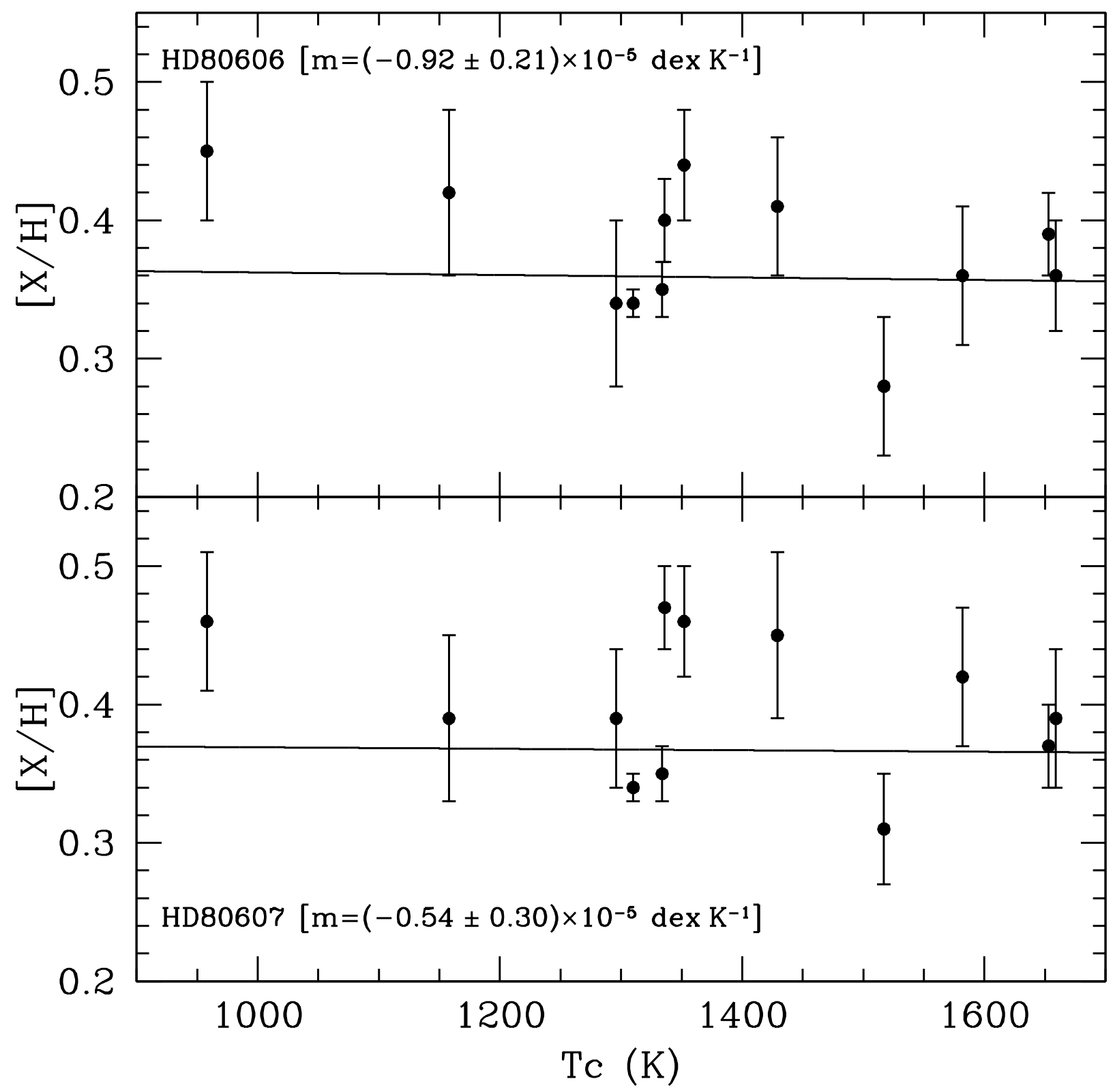

Fig. 4. - Weighted linear fits to abundances vs. condensation temperature $\left(T_{\mathrm{C}}\right)$ for HD 80606/07. 


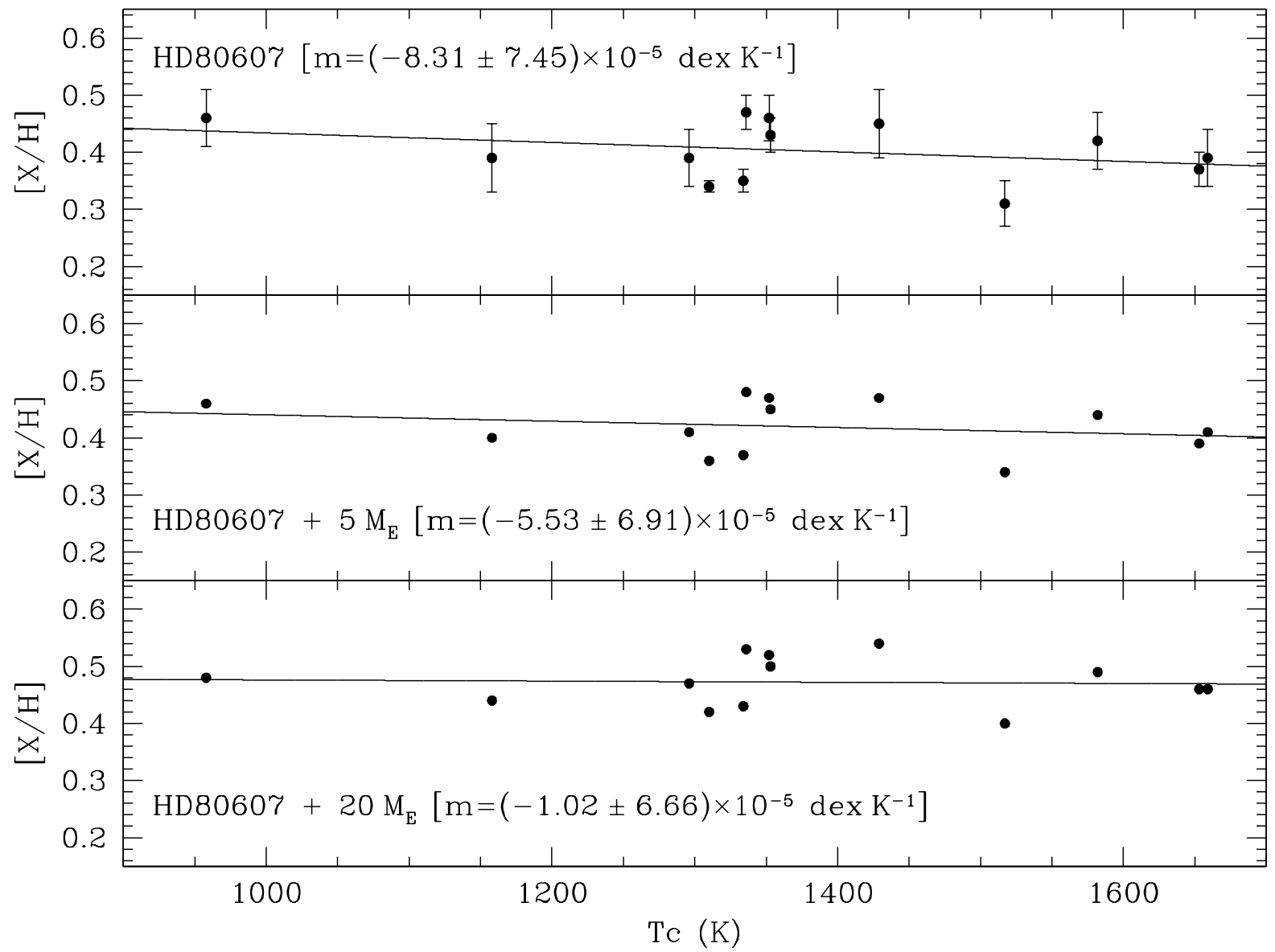

Fig. 5.- Top: Unweighted linear fit to HD 80607. Middle and Bottom: Unweighted linear fits to simulated abundances vs. condensation temperature $\left(T_{\mathrm{C}}\right)$ from our modeled accretion of $5 M_{\oplus}$ and $20 M_{\oplus}$ of material with Earth-like composition by a star like HD 80607. 
Table 5. HD 80606/07: Lines Measured, Equivalent Widths, and Abundances

\begin{tabular}{|c|c|c|c|c|c|c|c|c|c|c|c|c|}
\hline \multirow[b]{2}{*}{ Element } & \multirow{2}{*}{$\begin{array}{c}\lambda \\
(\AA)\end{array}$} & \multirow{2}{*}{$\begin{array}{c}\chi \\
(\mathrm{eV})\end{array}$} & \multirow[b]{2}{*}{$\log g f$} & \multirow[b]{2}{*}{$\mathrm{EW}_{\odot}$} & \multirow[b]{2}{*}{$\log N_{\odot}$} & \multirow[b]{2}{*}{$\log N_{\odot, \text { synth }}{ }^{\mathrm{a}}$} & \multicolumn{3}{|c|}{ HD 80606} & \multicolumn{3}{|c|}{ HD 80607} \\
\hline & & & & & & & EW & $\log N$ & $\log N_{\text {synth }}$ & EW & $\log N$ & $\log N_{\text {synth }}$ \\
\hline $\mathrm{Na} \mathrm{I}$ & 5682.63 & 2.10 & -0.700 & 100.9 & 6.30 & $\ldots$ & 142.9 & 6.70 & $\ldots$ & 146.0 & 6.70 & $\ldots$ \\
\hline $\mathrm{NaI}$ & 6154.23 & 2.10 & -1.560 & 38.4 & 6.31 & $\ldots$ & 76.5 & 6.77 & $\ldots$ & 80.4 & 6.80 & $\ldots$ \\
\hline $\mathrm{NaI}$ & 6160.75 & 2.10 & -1.260 & 56.1 & 6.28 & $\ldots$ & 100.6 & 6.78 & $\ldots$ & 102.1 & 6.77 & $\ldots$ \\
\hline $\mathrm{Mg} \mathrm{I}$ & 4730.03 & 4.35 & -2.523 & 67.3 & 7.83 & $\ldots$ & 103.0 & 8.23 & $\ldots$ & 110.2 & 8.30 & $\ldots$ \\
\hline $\mathrm{Al} \mathrm{I}$ & 6696.02 & 3.14 & -1.347 & 38.3 & 6.27 & $\ldots$ & 70.4 & 6.66 & $\ldots$ & 70.8 & 6.65 & $\ldots$ \\
\hline $\mathrm{Al} \mathrm{I}$ & 6698.67 & 3.14 & -1.647 & 22.9 & 6.27 & & 47.9 & 6.65 & $\ldots$ & 47.9 & 6.62 & $\ldots$ \\
\hline Si I & 5701.10 & 4.93 & -1.581 & 37.2 & 7.08 & $\ldots$ & 59.8 & 7.46 & $\ldots$ & 57.6 & 7.44 & $\ldots$ \\
\hline Si I & 5772.15 & 5.08 & -1.358 & 51.8 & 7.23 & $\ldots$ & 72.6 & 7.56 & $\ldots$ & 71.8 & 7.56 & $\ldots$ \\
\hline Si I & 6125.02 & 5.61 & -1.464 & 30.3 & 7.44 & $\ldots$ & 52.2 & 7.83 & $\ldots$ & 50.5 & 7.81 & $\ldots$ \\
\hline Si I & 6142.48 & 5.62 & -1.295 & 32.4 & 7.31 & $\ldots$ & 52.8 & 7.67 & $\ldots$ & 53.2 & 7.69 & $\ldots$ \\
\hline Si I & 6145.02 & 5.62 & -1.310 & 37.2 & 7.41 & $\ldots$ & 58.4 & 7.76 & $\ldots$ & 56.0 & 7.74 & $\ldots$ \\
\hline Si I & 6243.81 & 5.62 & -1.242 & 47.0 & 7.50 & $\ldots$ & 67.9 & 7.83 & $\ldots$ & 68.2 & 7.84 & $\ldots$ \\
\hline Si I & 6244.47 & 5.62 & -1.093 & 47.3 & 7.35 & & 67.5 & 7.67 & $\ldots$ & 67.7 & 7.68 & $\ldots$ \\
\hline Si I & 6414.98 & 5.87 & -1.035 & 51.5 & 7.55 & $\ldots$ & 71.2 & 7.83 & $\ldots$ & 70.3 & 7.82 & $\ldots$ \\
\hline Si I & 6741.63 & 5.98 & -1.428 & 15.2 & 7.35 & $\ldots$ & 29.1 & 7.72 & $\ldots$ & 29.0 & 7.72 & $\ldots$ \\
\hline Si I & 6848.58 & 5.86 & -1.524 & 17.5 & 7.41 & $\ldots$ & 32.7 & 7.78 & $\ldots$ & 30.6 & 7.76 & $\ldots$ \\
\hline Si I & 7405.77 & 5.61 & -0.313 & 97.9 & 7.19 & $\ldots$ & 115.8 & 7.44 & $\ldots$ & $\ldots$ & $\ldots$ & $\ldots$ \\
\hline $\mathrm{Ca} \mathrm{I}$ & 6161.30 & 2.52 & -1.266 & 63.5 & 6.30 & $\ldots$ & 90.5 & 6.62 & $\ldots$ & 92.7 & 6.64 & $\ldots$ \\
\hline $\mathrm{Ca} \mathrm{I}$ & 6166.44 & 2.52 & -1.142 & 67.2 & 6.24 & $\ldots$ & 93.6 & 6.54 & $\ldots$ & 96.0 & 6.56 & $\ldots$ \\
\hline $\mathrm{Ca} I$ & 6169.04 & 2.52 & -0.797 & 89.4 & 6.23 & $\ldots$ & 118.7 & 6.55 & $\ldots$ & 123.3 & 6.58 & $\ldots$ \\
\hline $\mathrm{Ca} \mathrm{I}$ & 6169.56 & 2.53 & -0.478 & 107.7 & 6.18 & $\ldots$ & 138.9 & 6.47 & $\ldots$ & 145.4 & 6.51 & $\ldots$ \\
\hline $\mathrm{Ca} \mathrm{I}$ & 6455.60 & 2.52 & -1.340 & 60.6 & 6.32 & $\ldots$ & 81.6 & 6.54 & $\ldots$ & 84.3 & 6.56 & $\ldots$ \\
\hline $\mathrm{Ca} \mathrm{I}$ & 6499.65 & 2.52 & -0.818 & 86.5 & 6.19 & $\ldots$ & 108.8 & 6.41 & $\ldots$ & 112.5 & 6.44 & $\ldots$ \\
\hline Sc II & 6245.64 & 1.51 & -1.030 & 34.7 & 3.07 & $\ldots$ & 48.9 & 3.44 & $\ldots$ & 49.4 & 3.49 & $\ldots$ \\
\hline Sc II & 6604.60 & 1.36 & -1.309 & 38.5 & 3.25 & $\ldots$ & 51.3 & 3.59 & $\ldots$ & 50.1 & 3.60 & $\ldots$ \\
\hline Ti I & 5022.87 & 0.83 & -0.434 & 69.5 & 4.74 & $\ldots$ & 94.2 & 5.04 & $\ldots$ & 101.2 & 5.17 & $\ldots$ \\
\hline Ti I & 5024.84 & 0.82 & -0.602 & 66.4 & 4.84 & $\ldots$ & 97.0 & 5.26 & $\ldots$ & 99.7 & 5.30 & $\ldots$ \\
\hline $\mathrm{Ti} \mathrm{I}$ & 5039.96 & 0.02 & -1.130 & 69.8 & 4.63 & $\ldots$ & 99.2 & 5.00 & $\ldots$ & 105.6 & 5.12 & $\ldots$ \\
\hline $\mathrm{Ti} \mathrm{I}$ & 5866.45 & 1.07 & -0.840 & 48.2 & 4.92 & $\ldots$ & 77.3 & 5.25 & $\ldots$ & 82.0 & 5.31 & $\ldots$ \\
\hline $\mathrm{Ti} \mathrm{I}$ & 6091.17 & 2.27 & -0.423 & 15.5 & 4.97 & $\ldots$ & 33.0 & 5.27 & $\ldots$ & 36.2 & 5.29 & $\ldots$ \\
\hline $\mathrm{Ti} \mathrm{I}$ & 6098.66 & 3.06 & -0.010 & 5.0 & 4.78 & $\ldots$ & 15.2 & 5.21 & $\ldots$ & 16.9 & 5.23 & $\ldots$ \\
\hline $\mathrm{Ti} \mathrm{I}$ & 6258.10 & 1.44 & -0.355 & 49.2 & 4.80 & $\ldots$ & 77.7 & 5.12 & $\ldots$ & 80.8 & 5.15 & $\ldots$ \\
\hline Ti II & 5336.79 & 1.58 & -1.590 & 68.5 & 4.78 & $\ldots$ & 81.0 & 5.13 & $\ldots$ & 78.9 & 5.14 & $\ldots$ \\
\hline Ti II & 5381.02 & 1.57 & -1.920 & 56.4 & 4.86 & $\ldots$ & 73.4 & 5.29 & $\ldots$ & 73.8 & 5.35 & $\ldots$ \\
\hline V I & 6081.44 & 1.05 & -0.579 & 14.0 & 3.88 & $\ldots$ & 38.8 & 4.29 & $\ldots$ & 43.2 & 4.32 & $\ldots$ \\
\hline V I & $6090.21^{\mathrm{c}}$ & 1.08 & -0.062 & 32.9 & 3.86 & 3.83 & 66.3 & 4.27 & 4.22 & 69.1 & 4.29 & 4.22 \\
\hline V I & $6111.65^{\mathrm{c}}$ & 1.04 & -0.715 & 10.7 & 3.86 & 3.81 & 37.5 & 4.39 & 4.26 & 41.3 & 4.41 & 4.29 \\
\hline V I & 6251.83 & 0.29 & -1.340 & 17.9 & 3.98 & $\ldots$ & 46.4 & 4.37 & $\ldots$ & 53.5 & 4.44 & $\ldots$ \\
\hline Cr I & 5702.31 & 3.45 & -0.667 & 23.1 & 5.78 & $\ldots$ & 49.1 & 6.19 & $\ldots$ & 53.4 & 6.25 & $\ldots$ \\
\hline $\mathrm{Cr} \mathrm{I}$ & 5783.06 & 3.32 & -0.500 & 35.9 & 5.76 & $\ldots$ & 55.2 & 6.01 & $\ldots$ & $\ldots$ & $\ldots$ & $\ldots$ \\
\hline Cr I & 5783.85 & 3.32 & -0.295 & 43.0 & 5.69 & $\ldots$ & 72.0 & 6.08 & $\ldots$ & 73.3 & 6.09 & $\ldots$ \\
\hline $\mathrm{Cr} \mathrm{I}$ & 5787.92 & 3.32 & -0.083 & 44.8 & 5.51 & $\ldots$ & 69.8 & 5.83 & $\ldots$ & 70.5 & 5.83 & $\ldots$ \\
\hline $\mathrm{Cr} \mathrm{I}$ & 7400.25 & 2.90 & -0.111 & 77.1 & 5.58 & $\ldots$ & 107.6 & 5.93 & $\ldots$ & 109.2 & 5.93 & $\ldots$ \\
\hline Mn I & $5432.55^{\mathrm{c}}$ & 0.00 & -3.795 & 46.1 & 5.38 & 5.27 & 43.5 & 5.37 & 5.21 & 111.1 & 6.25 & 5.66 \\
\hline $\mathrm{Fe} \mathrm{I}$ & 5322.04 & 2.28 & -2.800 & 57.6 & 7.20 & $\ldots$ & 80.6 & 7.52 & $\ldots$ & 81.0 & 7.54 & $\ldots$ \\
\hline $\mathrm{Fe} I$ & 5379.57 & 3.69 & -1.510 & 58.4 & 7.30 & $\ldots$ & 84.6 & 7.69 & $\ldots$ & 81.6 & 7.64 & $\ldots$ \\
\hline $\mathrm{Fe} \mathrm{I}$ & 5522.45 & 4.21 & -1.550 & 42.7 & 7.54 & $\ldots$ & 61.7 & 7.81 & $\ldots$ & 64.4 & 7.86 & $\ldots$ \\
\hline $\mathrm{Fe} I$ & 5543.94 & 4.22 & -1.140 & 59.5 & 7.43 & $\ldots$ & 82.9 & 7.77 & $\ldots$ & 85.4 & 7.81 & $\ldots$ \\
\hline Fe I & 5546.50 & 4.37 & -1.310 & 49.7 & 7.57 & $\ldots$ & 71.4 & 7.88 & $\ldots$ & 73.0 & 7.91 & $\ldots$ \\
\hline $\mathrm{Fe} I$ & 5546.99 & 4.22 & -1.910 & 27.9 & 7.61 & $\ldots$ & 55.7 & 8.07 & $\ldots$ & 57.7 & 8.10 & $\ldots$ \\
\hline $\mathrm{Fe} I$ & 5560.21 & 4.43 & -1.190 & 49.6 & 7.50 & $\ldots$ & 69.3 & 7.79 & $\ldots$ & 69.3 & 7.78 & $\ldots$ \\
\hline
\end{tabular}


Table 5-Continued

\begin{tabular}{|c|c|c|c|c|c|c|c|c|c|c|c|c|}
\hline \multirow[b]{2}{*}{ Element } & \multirow{2}{*}{$\begin{array}{c}\lambda \\
(\AA)\end{array}$} & \multirow{2}{*}{$\begin{array}{c}\chi \\
(\mathrm{eV})\end{array}$} & \multirow[b]{2}{*}{$\log g f$} & \multirow[b]{2}{*}{$\mathrm{EW}_{\odot}$} & \multirow[b]{2}{*}{$\log N_{\odot}$} & \multirow[b]{2}{*}{$\log N_{\odot, \text { synth }^{a}}{ }^{\mathrm{a}}$} & \multicolumn{3}{|c|}{ HD 80606} & \multicolumn{3}{|c|}{ HD 80607} \\
\hline & & & & & & & EW & $\log N$ & $\log N_{\text {synth }}$ & EW & $\log N$ & $\log N_{\text {synth }}$ \\
\hline $\mathrm{Fe} I$ & 5577.03 & 5.03 & -1.550 & 12.0 & 7.55 & $\ldots$ & 23.3 & 7.85 & $\ldots$ & 23.8 & 7.85 & $\ldots$ \\
\hline $\mathrm{Fe} I$ & 5579.34 & 4.23 & -2.400 & 11.1 & 7.61 & $\ldots$ & 24.5 & 7.96 & $\ldots$ & 24.1 & 7.94 & $\cdots$ \\
\hline $\mathrm{Fe} I$ & 5587.57 & 4.14 & -1.850 & 37.6 & 7.67 & $\ldots$ & 59.5 & 8.00 & $\ldots$ & 59.5 & 7.99 & $\ldots$ \\
\hline $\mathrm{Fe} I$ & 5651.47 & 4.47 & -2.000 & 18.5 & 7.70 & $\ldots$ & 34.9 & 8.02 & $\ldots$ & 35.5 & 8.02 & $\ldots$ \\
\hline $\mathrm{Fe} I$ & 5652.32 & 4.26 & -1.950 & 25.8 & 7.64 & $\ldots$ & 45.0 & 7.96 & $\cdots$ & 46.0 & 7.97 & $\ldots$ \\
\hline $\mathrm{Fe} I$ & 5661.35 & 4.28 & -1.740 & 21.6 & 7.34 & $\ldots$ & 41.5 & 7.70 & $\ldots$ & 42.4 & 7.71 & $\ldots$ \\
\hline $\mathrm{Fe} I$ & 5667.52 & 4.18 & -1.580 & 49.2 & 7.65 & $\ldots$ & 78.6 & 8.09 & $\ldots$ & 79.1 & 8.10 & $\cdots$ \\
\hline $\mathrm{Fe} I$ & 5677.68 & 4.10 & -2.700 & 6.5 & 7.52 & $\ldots$ & 15.8 & 7.89 & $\ldots$ & 16.6 & 7.90 & $\ldots$ \\
\hline $\mathrm{Fe} I$ & 5679.02 & 4.65 & -0.920 & 57.7 & 7.56 & $\ldots$ & 77.6 & 7.83 & $\ldots$ & 78.8 & 7.85 & $\cdots$ \\
\hline $\mathrm{Fe} I$ & 5680.24 & 4.19 & -2.580 & 9.8 & 7.68 & $\ldots$ & 22.7 & 8.05 & $\ldots$ & 24.0 & 8.07 & $\ldots$ \\
\hline $\mathrm{Fe} I$ & 5732.27 & 4.99 & -1.560 & 13.8 & 7.59 & $\ldots$ & 29.3 & 7.96 & $\ldots$ & 29.8 & 7.96 & $\ldots$ \\
\hline $\mathrm{Fe} I$ & 5741.85 & 4.26 & -1.850 & 31.0 & 7.65 & $\ldots$ & 51.8 & 7.97 & $\ldots$ & 52.9 & 7.98 & $\cdots$ \\
\hline $\mathrm{Fe} I$ & 5752.03 & 4.55 & -1.180 & 54.1 & 7.67 & $\ldots$ & 73.6 & 7.94 & $\ldots$ & 73.3 & 7.94 & $\ldots$ \\
\hline $\mathrm{Fe} I$ & 5775.08 & 4.22 & -1.300 & 55.8 & 7.52 & $\ldots$ & 79.4 & 7.86 & $\ldots$ & 79.6 & 7.86 & $\cdots$ \\
\hline $\mathrm{Fe} I$ & 5778.45 & 2.59 & -3.480 & 21.7 & 7.44 & $\ldots$ & 43.9 & 7.79 & $\ldots$ & 44.5 & 7.78 & $\ldots$ \\
\hline $\mathrm{Fe} I$ & 6079.00 & 4.65 & -1.120 & 43.8 & 7.52 & $\ldots$ & 64.3 & 7.81 & $\ldots$ & 66.7 & 7.85 & $\cdots$ \\
\hline $\mathrm{Fe} I$ & 6085.26 & 2.76 & -3.100 & 42.2 & 7.64 & $\ldots$ & 71.3 & 8.05 & $\ldots$ & 75.3 & 8.13 & $\ldots$ \\
\hline $\mathrm{Fe} I$ & 6098.24 & 4.56 & -1.880 & 16.4 & 7.58 & $\ldots$ & 36.0 & 7.99 & $\ldots$ & 33.2 & 7.92 & $\ldots$ \\
\hline $\mathrm{Fe} I$ & 6151.62 & 2.18 & -3.300 & 48.1 & 7.37 & $\ldots$ & 70.6 & 7.64 & $\ldots$ & 72.5 & 7.67 & $\ldots$ \\
\hline $\mathrm{Fe} \mathrm{I}$ & 6159.37 & 4.61 & -1.970 & 12.3 & 7.57 & $\ldots$ & 27.3 & 7.95 & $\ldots$ & 29.2 & 7.98 & $\ldots$ \\
\hline $\mathrm{Fe} I$ & 6165.36 & 4.14 & -1.470 & 43.6 & 7.38 & $\ldots$ & 65.5 & 7.69 & $\ldots$ & 65.4 & 7.69 & $\cdots$ \\
\hline $\mathrm{Fe} I$ & 6187.99 & 3.94 & -1.720 & 46.2 & 7.48 & $\ldots$ & 71.6 & 7.85 & $\ldots$ & 73.3 & 7.88 & $\ldots$ \\
\hline $\mathrm{Fe} I$ & 6220.78 & 3.88 & -2.460 & 18.6 & 7.57 & $\ldots$ & 37.3 & 7.92 & $\cdots$ & 39.8 & 7.96 & $\cdots$ \\
\hline $\mathrm{Fe} I$ & 6226.73 & 3.88 & -2.220 & 28.3 & 7.57 & $\ldots$ & 54.7 & 8.00 & $\ldots$ & 51.2 & 7.93 & $\ldots$ \\
\hline $\mathrm{Fe} I$ & 6229.23 & 2.85 & -2.810 & 37.7 & 7.35 & $\ldots$ & 64.1 & 7.72 & $\ldots$ & 62.4 & 7.68 & $\ldots$ \\
\hline $\mathrm{Fe} I$ & 6240.65 & 2.22 & -3.230 & 47.7 & 7.32 & $\ldots$ & 75.8 & 7.69 & $\ldots$ & 75.2 & 7.68 & $\cdots$ \\
\hline $\mathrm{Fe} I$ & 6380.74 & 4.19 & -1.380 & 52.2 & 7.48 & $\ldots$ & 77.1 & 7.83 & $\ldots$ & 72.6 & 7.76 & $\ldots$ \\
\hline $\mathrm{Fe} I$ & 6608.02 & 2.28 & -4.030 & 17.1 & 7.50 & $\ldots$ & 37.4 & 7.85 & $\ldots$ & 39.2 & 7.86 & $\ldots$ \\
\hline $\mathrm{Fe} I$ & 6609.11 & 2.56 & -2.690 & 65.1 & 7.40 & $\ldots$ & 90.9 & 7.74 & $\ldots$ & 92.1 & 7.76 & $\ldots$ \\
\hline $\mathrm{Fe} I$ & 6627.54 & 4.55 & -1.680 & 29.4 & 7.68 & $\ldots$ & 52.3 & 8.05 & $\ldots$ & 50.3 & 8.01 & $\cdots$ \\
\hline $\mathrm{Fe} I$ & 6653.85 & 4.15 & -2.520 & 10.9 & 7.60 & $\ldots$ & 21.3 & 7.88 & $\ldots$ & 24.1 & 7.93 & $\ldots$ \\
\hline $\mathrm{Fe} I$ & 6703.57 & 2.76 & -3.160 & 36.2 & 7.55 & $\ldots$ & 62.4 & 7.90 & $\ldots$ & 60.1 & 7.85 & $\ldots$ \\
\hline $\mathrm{Fe} I$ & 6710.32 & 1.49 & -4.880 & 18.8 & 7.61 & $\ldots$ & 42.1 & 7.96 & $\ldots$ & 40.6 & 7.90 & $\ldots$ \\
\hline $\mathrm{Fe} I$ & 6713.74 & 4.80 & -1.600 & 20.2 & 7.61 & $\ldots$ & 40.5 & 8.00 & $\ldots$ & 39.2 & 7.97 & $\ldots$ \\
\hline $\mathrm{Fe} I$ & 6716.22 & 4.58 & -1.920 & 15.5 & 7.58 & $\ldots$ & 31.4 & 7.93 & $\ldots$ & 30.9 & 7.91 & $\ldots$ \\
\hline $\mathrm{Fe} I$ & 6725.35 & 4.10 & -2.300 & 17.4 & 7.56 & $\ldots$ & 34.7 & 7.90 & $\ldots$ & 36.5 & 7.92 & $\ldots$ \\
\hline $\mathrm{Fe} I$ & 6726.67 & 4.61 & -1.130 & 45.8 & 7.49 & $\ldots$ & 71.0 & 7.86 & $\cdots$ & 69.3 & 7.83 & $\cdots$ \\
\hline $\mathrm{Fe} I$ & 6733.15 & 4.64 & -1.580 & 26.4 & 7.59 & $\ldots$ & 49.4 & 7.98 & $\ldots$ & 48.4 & 7.95 & $\ldots$ \\
\hline $\mathrm{Fe} I$ & 6739.52 & 1.56 & -4.790 & 11.8 & 7.35 & $\ldots$ & 27.8 & 7.67 & $\ldots$ & 30.3 & 7.69 & $\ldots$ \\
\hline $\mathrm{Fe} I$ & 6745.09 & 4.58 & -2.160 & 8.6 & 7.53 & $\ldots$ & 19.0 & 7.87 & $\ldots$ & 19.2 & 7.86 & $\ldots$ \\
\hline $\mathrm{Fe} I$ & 6745.96 & 4.08 & -2.770 & 7.6 & 7.60 & $\ldots$ & 16.1 & 7.90 & $\ldots$ & 17.4 & 7.93 & $\ldots$ \\
\hline $\mathrm{Fe} \mathrm{I}$ & 6752.72 & 4.64 & -1.300 & 34.6 & 7.49 & $\ldots$ & 59.1 & 7.86 & $\ldots$ & 58.3 & 7.85 & $\ldots$ \\
\hline Fe II & 5197.58 & 3.23 & -2.348 & 77.1 & 7.32 & $\ldots$ & 86.7 & 7.69 & $\ldots$ & 80.0 & 7.63 & $\ldots$ \\
\hline $\mathrm{Fe}$ II & 5234.62 & 3.22 & -2.279 & 77.8 & 7.26 & $\ldots$ & 88.5 & 7.64 & $\ldots$ & 86.9 & 7.69 & $\ldots$ \\
\hline $\mathrm{Fe}$ II & 5414.07 & 3.22 & -3.645 & 28.6 & 7.56 & $\ldots$ & 32.4 & 7.80 & $\ldots$ & 31.7 & 7.84 & $\ldots$ \\
\hline Fe II & 6084.11 & 3.20 & -3.881 & 20.0 & 7.53 & $\ldots$ & 27.3 & 7.89 & $\ldots$ & 25.6 & 7.89 & $\ldots$ \\
\hline $\mathrm{Fe} I I$ & 6113.32 & 3.22 & -4.230 & 12.5 & 7.64 & $\ldots$ & 16.8 & 7.94 & $\ldots$ & 17.6 & 8.01 & $\ldots$ \\
\hline Fe II & 6149.26 & 3.89 & -2.841 & 34.5 & 7.51 & $\ldots$ & 45.6 & 7.93 & $\ldots$ & 39.1 & 7.85 & $\ldots$ \\
\hline Fe II & 6239.95 & 3.89 & -3.573 & 13.3 & 7.63 & $\ldots$ & 19.3 & 8.00 & $\ldots$ & 17.8 & 8.00 & $\ldots$ \\
\hline Fe II & 6247.56 & 3.89 & -2.435 & 51.2 & 7.46 & $\ldots$ & 58.5 & 7.80 & $\ldots$ & 54.5 & 7.79 & $\ldots$ \\
\hline Co I & $5301.04^{\mathrm{c}}$ & 1.71 & -2.000 & 19.9 & 4.96 & $\ldots$ & 5.9 & 4.25 & 5.40 & 50.4 & 5.47 & 5.40 \\
\hline Co I & 5647.23 & 2.28 & -1.560 & 14.9 & 4.90 & $\ldots$ & 35.7 & 5.32 & $\ldots$ & 37.8 & 5.35 & $\ldots$ \\
\hline
\end{tabular}


Table 5-Continued

\begin{tabular}{|c|c|c|c|c|c|c|c|c|c|c|c|c|}
\hline \multirow[b]{2}{*}{ Element } & \multirow{2}{*}{$\begin{array}{c}\lambda \\
(\AA)\end{array}$} & \multirow{2}{*}{$\begin{array}{c}\chi \\
(\mathrm{eV})\end{array}$} & \multirow[b]{2}{*}{$\log g f$} & \multirow[b]{2}{*}{$\mathrm{EW} \odot$} & \multirow[b]{2}{*}{$\log N_{\odot}$} & \multirow[b]{2}{*}{$\log N_{\odot, \text { synth }^{a}}$} & \multicolumn{3}{|c|}{ HD 80606} & \multicolumn{3}{|c|}{ HD 80607} \\
\hline & & & & & & & EW & $\log N$ & $\log N_{\text {synth }}$ & EW & $\log N$ & $\log N_{\text {synth }}$ \\
\hline Co I & 6093.14 & 1.74 & -2.440 & 8.5 & 4.94 & $\ldots$ & 26.7 & 5.43 & $\ldots$ & $\ldots$ & $\ldots$ & $\ldots$ \\
\hline Co I & 6678.80 & 1.96 & -2.680 & 6.3 & 5.22 & $\ldots$ & 17.7 & 5.63 & $\ldots$ & 20.5 & 5.68 & $\ldots$ \\
\hline Co I & $6814.94^{\mathrm{c}}$ & 1.96 & -1.900 & 18.8 & 4.97 & $\ldots$ & 49.5 & 5.50 & 5.42 & 51.5 & 5.52 & 5.46 \\
\hline $\mathrm{Ni} \mathrm{I}$ & 5748.35 & 1.68 & -3.260 & 27.7 & 6.18 & $\ldots$ & 54.4 & 6.61 & $\ldots$ & 55.7 & 6.63 & $\ldots$ \\
\hline $\mathrm{Ni} \mathrm{I}$ & 5754.65 & 1.94 & -2.330 & 75.9 & 6.43 & $\ldots$ & 105.4 & 6.90 & $\ldots$ & 107.3 & 6.95 & $\ldots$ \\
\hline $\mathrm{Ni} \mathrm{I}$ & 5760.83 & 4.11 & -0.800 & 34.1 & 6.30 & $\ldots$ & 55.6 & 6.59 & $\ldots$ & 54.5 & 6.58 & $\ldots$ \\
\hline $\mathrm{Ni} I$ & 5846.99 & 1.68 & -3.210 & 22.2 & 6.00 & $\ldots$ & 47.6 & 6.43 & $\ldots$ & 48.7 & 6.44 & $\ldots$ \\
\hline $\mathrm{Ni} I$ & 6111.07 & 4.09 & -0.870 & 34.0 & 6.26 & $\ldots$ & 60.2 & 6.72 & $\ldots$ & 59.6 & 6.71 & $\ldots$ \\
\hline $\mathrm{Ni} \mathrm{I}$ & 6128.96 & 1.68 & -3.330 & 25.8 & 6.19 & $\ldots$ & 50.0 & 6.57 & $\ldots$ & 51.0 & 6.58 & $\ldots$ \\
\hline $\mathrm{Ni} \mathrm{I}$ & 6130.13 & 4.27 & -0.960 & 21.4 & 6.23 & $\ldots$ & 40.0 & 6.61 & $\ldots$ & 40.3 & 6.62 & $\ldots$ \\
\hline $\mathrm{Ni} I$ & 6133.96 & 4.09 & -1.830 & 4.9 & 6.20 & $\ldots$ & 13.4 & 6.65 & $\ldots$ & 14.2 & 6.68 & $\ldots$ \\
\hline $\mathrm{Ni} \mathrm{I}$ & 6175.36 & 4.09 & -0.559 & 47.6 & 6.20 & $\ldots$ & 68.6 & 6.55 & $\ldots$ & 70.2 & 6.59 & $\ldots$ \\
\hline $\mathrm{Ni} \mathrm{I}$ & 6176.81 & 4.09 & -0.260 & 61.8 & 6.15 & & 86.4 & 6.54 & $\ldots$ & 89.1 & 6.59 & $\ldots$ \\
\hline $\mathrm{Ni} I$ & 6177.24 & 1.83 & -3.500 & 15.4 & 6.22 & $\ldots$ & 33.9 & 6.60 & $\ldots$ & 36.0 & 6.63 & $\ldots$ \\
\hline $\mathrm{Ni} I$ & 6186.71 & 4.11 & -0.960 & 31.1 & 6.30 & $\ldots$ & 52.0 & 6.67 & $\ldots$ & 52.8 & 6.69 & $\ldots$ \\
\hline $\mathrm{Ni} \mathrm{I}$ & 6223.98 & 4.11 & -0.910 & 28.4 & 6.20 & $\ldots$ & 51.4 & 6.61 & $\ldots$ & 50.2 & 6.60 & $\ldots$ \\
\hline $\mathrm{Ni} \mathrm{I}$ & 6230.09 & 4.11 & -1.260 & 22.4 & 6.40 & $\ldots$ & 42.4 & 6.80 & $\ldots$ & 44.1 & 6.83 & $\ldots$ \\
\hline $\mathrm{Ni} \mathrm{I}$ & 6327.59 & 1.68 & -3.150 & 40.0 & 6.28 & $\ldots$ & 65.4 & 6.65 & $\ldots$ & 70.1 & 6.74 & $\ldots$ \\
\hline $\mathrm{Ni} \mathrm{I}$ & 6370.34 & 3.54 & -1.940 & 12.7 & 6.23 & $\ldots$ & 30.7 & 6.69 & $\ldots$ & 30.7 & 6.69 & $\ldots$ \\
\hline $\mathrm{Ni} \mathrm{I}$ & 6378.25 & 4.15 & -0.830 & 34.5 & 6.28 & $\ldots$ & 55.0 & 6.63 & $\ldots$ & 57.2 & 6.68 & $\ldots$ \\
\hline $\mathrm{Ni}$ I & 6643.63 & 1.68 & -2.300 & 93.6 & 6.37 & $\ldots$ & 124.0 & 6.78 & $\ldots$ & 128.2 & 6.86 & $\ldots$ \\
\hline $\mathrm{Ni}$ I & 6767.77 & 1.83 & -2.170 & 76.5 & 6.08 & $\ldots$ & 104.8 & 6.48 & $\ldots$ & 106.4 & 6.53 & $\ldots$ \\
\hline
\end{tabular}

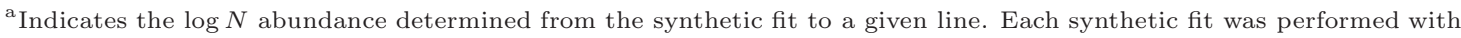
the MOOG synth driver. Synthetic fits were only performed for the subset of V, Mn, and Co lines that were tested for hfs.

${ }^{\mathrm{c}}$ Indicates that the spectral line was tested for hfs. 